Check for updates

Cite this: Phys. Chem. Chem. Phys., 2017, 19, 26201

Received 20th July 2017.

Accepted 7th September 2017

DOI: $10.1039 / \mathrm{c} 7 \mathrm{cp} 04914 \mathrm{e}$

rsc.li/pccp

\section{Redistribution of valence and conduction band states depending on the method of modification of $\mathrm{SiO}_{2}$ structure}

\author{
A. S. Konashuk (D) and E. O. Filatova (D)*
}

\begin{abstract}
The effect of introducing porosity and the insertion of methyl groups in $\mathrm{SiO}_{4}$ tetrahedra on the distribution of valence and conduction band states of $\mathrm{SiO}_{2}$ was studied using high-resolution near edge X-ray absorption fine structure spectroscopy (NEXAFS) and soft X-ray photoelectron spectroscopy (XPS). Alignment of NEXAFS spectra and valence band XPS spectra in a common energy scale was performed using binding energies of the initial levels obtained by XPS. It was established that the insertion of methyl groups into $\mathrm{SiO}_{4}$ tetrahedra leads to a significant shift of the top of the valence band $\mathrm{E}_{\mathrm{V}}$ to smaller binding energies due to the reduction of the electronegativity of the nearest surrounding neighbors of the $\mathrm{Si}$ atoms, while introducing porosity changes the position of $E_{V}$ only slightly. The position of the bottom of the conduction band is affected by neither the introduction of porosity nor the insertion of methyl groups. The formation of the $\pi^{\star} \mathrm{C}=\mathrm{C}$ state near the Fermi level, caused by the presence of porogen residues in the structure of organosilicate glass (OSG) and responsible for the leakage currents, was revealed. It was found that high level porosity in OSG films induces a significant variation of $\mathrm{Si}-\mathrm{O}-\mathrm{Si}$ valence angles. A number of $\mathrm{Si}-\mathrm{O}$ dangling bonds were found in the surface layers of por- $\mathrm{SiO}_{2}$, while methyl groups effectively passivated these dangling bonds in OSG films. The obtained results are important for understanding the regularities of electronic structure formation in $\mathrm{SiO}_{2}$-based low- $k$ dielectrics, which is necessary for the reduction of energy dissipated in semiconductor integrated circuits (ICS).
\end{abstract}

\section{Introduction}

The packing of a microchip becomes denser every year and the greater part of its cross-section gets occupied by metallic lines. ${ }^{1}$ The insulation of metallic lines from one another has become challenging due to such small distances between them that a parasitic capacity can lead to significant RC delays, dynamic power consumption and parasitic cross-talking. ${ }^{1,2}$ In order to reduce the parasitic capacity, one needs to decrease the dielectric permittivity value $(k)$ of the insulating material. The most reasonable method is to use $\mathrm{SiO}_{2}$, which is traditional for microelectronics, and modify its structure to develop low- $k$ dielectrics based on $\mathrm{SiO}_{2}$. Generally, there are two ways of decreasing the $k$-value, which are already being used in the industry: (1) introducing porosity into the structure of $\mathrm{SiO}_{2}$ (decreasing density and consequently permittivity) and (2) substitution of some oxygen atoms by terminating $-\mathrm{CH}_{3}$ methyl groups (decreasing the polarizability of bonds and also decreasing the density to some extent). Materials obtained in such way are referred to as organosilicate glass (OSG). The significant modification of the structure of $\mathrm{SiO}_{2}$ may

St. Petersburg State University, Ul'yanovskaya Str. 1, Peterhof, 198504,

St. Petersburg, Russia. E-mail: elenaofilatova@mail.ru; Tel: +7 8124284352 significantly alter its electronic structure, which would without a doubt affect the functionality of the insulating layer in a microchip. The insertion of methyl groups should lead to the redistribution of effective charge on $\mathrm{Si}$ atoms due to the lower electronegativity of carbon. As was shown previously, ${ }^{3}$ the redistribution of effective charge causes a significant offset of the bottom of the conduction band. Introducing porosity may be accompanied by the formation of $\mathrm{C}=\mathrm{C}$ clusters, which induces parasitic states overlapping with the Fermi energies of common electrode materials. ${ }^{4}$ Moreover, a number of defects (particularly dangling bonds) may be developed. ${ }^{5}$ In view of this, a joint study of the redistribution of valence and conduction band states, depending on the method of modification of $\mathrm{SiO}_{2}$ structure, is critically important.

In the present work we report the results obtained for the valence and conduction band states of $\alpha$-quartz, amorphous $\mathrm{SiO}_{2}$, porous $\mathrm{SiO}_{2}$ and OSG films with/without carbon clusters. These results were obtained by means of two independent X-ray spectroscopic techniques, in particular high-resolution near edge X-ray absorption fine structure spectroscopy (NEXAFS) and X-ray photoelectron spectroscopy (XPS), which have become powerful tools for probing empty and occupied electronic states, respectively. All the measurements were performed jointly in 
identical experimental conditions. NEXAFS spectroscopy provides information about the local (associated with hole localization in the core shell) and partial (allowing for certain angular momentum symmetry) electronic density of states of the conduction band. In photoelectron spectroscopy, the electrons from the valence band and core levels are excited in a continuous spectrum at fixed excitation photon energy.

The goal of the current study is the careful analysis of the energy positions of the top of the valence band $\left(\mathrm{E}_{\mathrm{V}}\right)$ and the bottom of the conduction band $\left(\mathrm{E}_{\mathrm{C}}\right)$, depending on the introduction of porosity and the insertion of methyl groups into the structure of $\mathrm{SiO}_{2}$. In order to gain further insight into the electronic structure of differently modified $\mathrm{SiO}_{2}$, the $\mathrm{Si}_{2,3^{-}}$, $\mathrm{C} \mathrm{K}$ - and $\mathrm{O} \mathrm{K}$-absorption spectra were aligned on a common energy scale, using the value of energy separation between the $\mathrm{O}$ 1s, C 1s and Si 2p core levels derived from XPS measurement.

\section{Experimental}

Natural monocrystal of $\alpha$-quartz (0001) (6H hexagonal with parameters $a=4.913 \AA$, $c=5.405 \AA$ and $d_{003}=1.801 \AA$ ) was used as crystalline $\mathrm{SiO}_{2}$. Amorphous $\mathrm{SiO}_{2}\left(\mathrm{am}-\mathrm{SiO}_{2}\right)$ was grown via dry oxidation of $\mathrm{p}$-Si (100) wafer at $1050{ }^{\circ} \mathrm{C}$ in $\mathrm{O}_{2}$; the thickness of the obtained film was greater than $100 \mathrm{~nm}$. The porous $\mathrm{SiO}_{2}$ (por-SiO ${ }_{2}$ ) was synthesized on $\mathrm{Si}$ substrates by the "spin-on"-technique 6 from the liquid phase (silica sol), followed by drying in an infrared furnace. Low- $k$ OSG layers were grown on p-Si(100)/ $\mathrm{SiO}_{2}(1 \mathrm{~nm})$ substrates by two methods: (i) selfassembly technology (SAT) $(k=2,0)$ (without porogen) using polymer templates; (ii) conventional plasma enhanced chemical vapor deposition (PECVD-1) $(k=2,3)$, followed by the removal of sacrificial $\mathrm{C}_{x} \mathrm{H}_{y}$ porogens using thermal-UV curing at $430{ }^{\circ} \mathrm{C}$. The SAT low- $k$ film was formed by condensing a hydrolyzed alkylated silica sol in the presence of a polymeric surfactant. This surfactant acts as a template to produce a regular porous structure as the film dries. In this method, the surfactant acts as a porogen and evaporates during thermal curing $\left(400{ }^{\circ} \mathrm{C}\right)$, leaving behind a porous silica network with alkyl groups passivating the internal and external surfaces of the film. ${ }^{7}$ The PECVD- 1 film was deposited at $300{ }^{\circ} \mathrm{C}$ from the organosilane matrix precursor and sacrificial organic porogen and subsequently cured as described in detail elsewhere. ${ }^{8}$ The parameters of all porous samples are summarized in Table 1.

The samples were jointly studied using NEXAFS and XPS methods in identical experimental conditions. NEXAFS and XPS measurements were performed at the RGL-station on the Russian-German beamline at the BESSY II synchrotron light source of the Helmholtz-Zentrum, Berlin. ${ }^{9}$ The XPS spectra of

Table 1 The values of average pore diameter, porosity degree and thickness of por-SiO 2, PECVD-1 and SAT samples

\begin{tabular}{llll}
\hline Sample & Pore diameter $\mathrm{nm}$ & Porosity degree (\%) & Thickness (nm) \\
\hline por-SiO $_{2}$ & 2 & 15 & 200 \\
PECVD-1 & 4 & 30 & 180 \\
SAT & 5 & 40 & 180
\end{tabular}

core levels were taken at the excitation photon energy of $700 \mathrm{eV}$ and that of the valence band at $120 \mathrm{eV}$, using a hemispherical electron energy analyzer (Specs Phoibos 150). All the photoemission spectra were collected with the analyzer and monochromator energy resolution better than $430 \mathrm{meV}$. The binding energy scale was referenced to the value of the $\mathrm{Au} 4 \mathrm{f}_{7 / 2}$ photoelectron peak position $(83.95 \mathrm{eV}) .^{10,11}$ The charge neutralization system was used in order to neutralize charging during photon excitation. The XPS spectra of all the studied samples were measured without surface cleaning. Such an approach was caused by the high sensitivity of OSG to any surface treatment, which can alternate the structure of the OSG surface layers (specifically cleaving $\mathrm{Si}-\mathrm{CH}_{3}$ bonds). ${ }^{12,13}$ Nevertheless, all the XPS spectra were additionally measured for OSG films and $\mathrm{am}-\mathrm{SiO}_{2}$ after short portions of $\mathrm{Ar}^{+}$ion sputtering at a $30^{\circ}$ sputtering angle (from the surface), using $200 \mathrm{eV}$ kinetic energy of ions. The spectra of OSG were indistinguishable, while the $\mathrm{C} 1 \mathrm{~s}$ line of $\mathrm{am}_{-} \mathrm{SiO}_{2}$ was gradually decreased after each step of sputtering: first, the sputtering conditions used actually provided mild cleaning of the surface; second, the structure of OSG was not affected by the ion sputtering and the surface was free of any adhesion layer, even before $\mathrm{Ar}^{+}$ion sputtering. This fact confirms the accuracy of the obtained C 1s XPS and also CK-absorption spectra corresponding to the distribution of electronic states of OSG films without contribution of an adhesion layer.

NEXAFS spectra were measured at the incident angle of $45^{\circ}$ in the vicinity of $\mathrm{Si}_{2,3^{-}}, \mathrm{C} \mathrm{K}$ - and $\mathrm{O} \mathrm{K}$-absorption edges, with energy resolution better than $E / \Delta E=3500$. The spectra were obtained by monitoring the total electron yield from the samples when the drain current from the sample was measured. All the spectra were normalized to the continuum jump after the subtraction of the linear background extrapolated from the energy region below the corresponding absorption onset. Calibration of the photon energy scale was performed by measurement of the $\mathrm{Au} 4 \mathrm{f}_{7 / 2}$ photoelectron peak with photon excitation in the 1st and 2nd orders of diffraction. The actual photon energies were equated to the difference between the 2nd order and 1 st order $\mathrm{Au} 4 \mathrm{f}_{7 / 2}$ kinetic energies.

Also the XPS spectra were measured at the Resource Center of "Physical Methods of Surface Investigation" SPbSU using a Thermo Fisher Scientific Escalab 250Xi spectrometer with monochromated AlK $\alpha$ excitation $(1486.6 \mathrm{eV})$; the energy resolution was about $0.3 \mathrm{eV}$. The charge neutralization system was used in order to neutralize charging during photon excitation. Greater photon energy allowed the XPS spectra to be obtained from considerably greater depth ( 2 and 6 times greater for the core levels and valence band, respectively), according to ref. 14 . The energy positions of the core levels were the same as for those measured at BESSY II.

To control the shape of the CK-absorption spectra of OSG films, the CK spectra were measured using X-ray reflection spectroscopy. The X-ray reflection spectra were measured at grazing incidence angles $2^{\circ}$ and $4^{\circ}$. The measurement was performed at the reflectometer station at the Optics beamline ${ }^{15}$ at BESSY II. Changing the grazing incidence angle allows one to change the depth of formation of reflected radiation and obtain information from 
different probing depths. ${ }^{16}$ The CK-absorption spectra were calculated from measured reflection spectra using the KramersKronig relations as described in the previous publications. ${ }^{17,18}$ The CK-absorption spectra were almost indistinguishable, which further confirms that the obtained CK-absorption spectra correspond to the distribution of electronic states of OSG films without contribution of an adhesion layer.

\section{Results and discussion}

To understand the redistribution of valence and conduction band states, depending on methods of modification of $\mathrm{SiO}_{2}$ structure, we have analyzed together $\alpha$-quartz, am-SiO ${ }_{2}$, por-SiO $\mathrm{S}_{2}$ and OSG films (SAT, PECVD-1), since in general, the main structural units of $\mathrm{SiO}_{2}$ compounds and OSG are $\mathrm{SiO}_{4}$ and $\mathrm{SiO}_{4-n}\left(\mathrm{CH}_{3}\right)_{n}$ tetrahedra, respectively. Fig. 1 shows the $\operatorname{SiL}_{2,3}(2 \mathrm{p})$-absorption spectra of all the mentioned compounds measured by total electron yield. We first of all gave consideration to the $\mathrm{SiL}_{2,3}$-absorption spectra of $\alpha$-quartz, am-SiO ${ }_{2}$ and por-SiO ${ }_{2}$. As observed from Fig. 1, the spectra correlate well in number and energy position with the main features $(a, b$ and $c$ ) of the fine structure. In the quasi-molecular approach, the fine structure features $a, b$ and $c$ originate from transitions from $\mathrm{Si} 2 \mathrm{p}$-states to the $\left[\mathrm{SiO}_{4}\right]^{-5}$ cluster excited states, with $a_{1}$ (Si 3s-like), $t_{2}$ (Si 3p-like) and $e$ (Si d-like) symmetry, respectively ${ }^{19,20}$ (the states of silicon ion majorly contributing to the wave functions of the molecular states are given in brackets). The presence of the double feature $a-a^{\prime}$ is due to spin-orbit splitting of the initial Si 2p level, which is equal to $0.6 \mathrm{eV}$. Note that feature $c$ is associated with transitions to the $e$-continuum states and reflects the influence of the nearest surrounding neighbors (the molecular field of the oxygen atoms) on the silicon atom photoabsorption process. In turn, the $c^{\prime}$ and $c^{\prime \prime}$ features in the $\mathrm{SiL}_{2,3}$-absorption spectrum of $\alpha$-quartz reflect the presence of the long-range order in the $\alpha$-quartz crystal. ${ }^{20}$ Consistent with this interpretation, these features virtually disappear in the spectrum of the $\mathrm{am}_{-} \mathrm{SiO}_{2}$ due to the lack of longrange order in its structure.

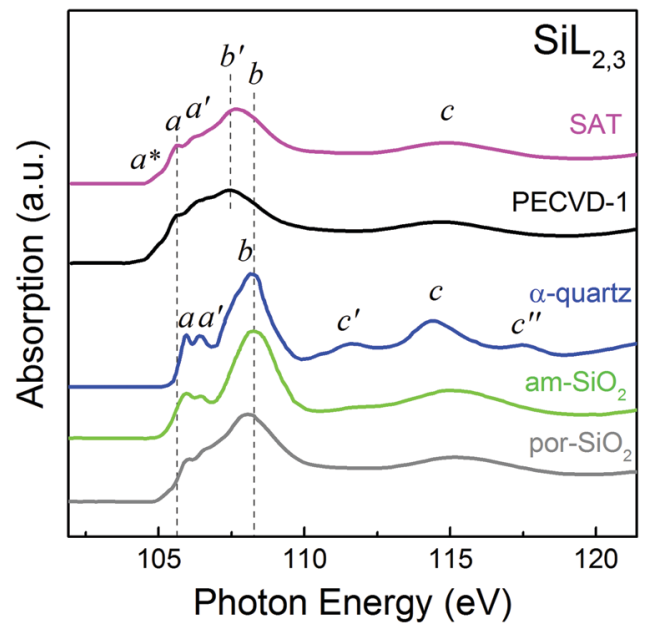

Fig. $1 \mathrm{SiL}_{2,3}(2 \mathrm{p})$-absorption spectra of $\alpha$-quartz, am- $\mathrm{SiO}_{2}$, por- $\mathrm{SiO}_{2}, \mathrm{SAT}$ and PECVD-1 films measured by monitoring the total electron yield when the drain current from the sample is measured.
Combined analysis of the $\mathrm{SiL}_{2,3}$-absorption spectra in the sequence of $\alpha$-quartz to am-SiO ${ }_{2}$, and further to por-SiO ${ }_{2}$, reveals a significant broadening of the feature $b$. In the por-SiO $\mathrm{S}_{2}$ structure, the additional broadening might arise due to the porosity of its structure, which leads to further network distortion. Despite the general similarity of these spectra, an appreciable decrease in the peak intensity $a$ and a significant reduction of the contrast of the doublet structure $a-a^{\prime}$ can be additionally traced in the spectrum of por-SiO $\mathrm{S}_{2}$. The illegibility of the structure $a-a^{\prime}$ indicates a violation of the crystal structure of por-SiO ${ }_{2}$ due to the existence of a considerable number of dangling $\mathrm{Si}-\mathrm{O}$ bonds and tetrahedra $\mathrm{SiO}_{4}$ distorted to varying degrees. In this case, there is a significant energy dispersion of the transitions from the $\mathrm{Si} 2 \mathrm{p}$ level to the unoccupied MO's for different silicon atoms, which as a consequence leads to the "slurring" of the structure in the $\mathrm{SiL}_{2,3}$ absorption spectra. Additional evidence of this conjecture can be found in the pre-edge region of the $\mathrm{SiL}_{2,3}$-absorption spectra. The joint analysis of the pre-edge region of all the spectra (Fig. 1) indicates the appearance of the shoulder $a^{*}$ in the spectra of the am-SiO ${ }_{2}$ and por-SiO ${ }_{2}$, which is very likely connects with the presence of dangling $\mathrm{Si}-\mathrm{O}$ bonds. Clearly, por- $\mathrm{SiO}_{2}$ has more dangling $\mathrm{Si}-\mathrm{O}$ bonds compared to $\mathrm{am}-\mathrm{SiO}_{2}$. One can conclude that the occurrence of porosity in the structure of $\mathrm{SiO}_{2}$ is displayed in the $\mathrm{SiL}_{2,3}$-absorption spectrum through the general lowering of the fine structure contrast. Also, a minor $(0.2 \mathrm{eV})$ low energy shift of feature $b$ in the spectrum of por-SiO ${ }_{2}$ is traced.

Joint analysis of the $\mathrm{SiL}_{2,3}$-absorption spectra (Fig. 1) of SAT, PECVD-1, $\alpha$-quartz, am- $\mathrm{SiO}_{2}$ and por-SiO ${ }_{2}$ revealed the general similarity of the OSG films spectra, compared to por-SiO $\mathrm{S}_{2}$. One can expect that the studied OSG-films have a nanoporous structure formed from amorphous silica-like matrix with a considerable number of pristine $\mathrm{SiO}_{4}$ tetrahedra. At the same time, some additional changes in the $\mathrm{SiL}_{2,3}$-absorption spectra of OSG films can be traced.

In the spectra of OSG films, the shape of the second main feature $b^{\prime}-b$ is quite asymmetric, with the shoulder coming from the higher energy side. Furthermore, the main peak $b^{\prime}$ is seen to be shifted by $0.5 \mathrm{eV}$ toward lower energy, relative to peak $b$ in the spectra of $\alpha$-quartz and am- $\mathrm{SiO}_{2}$, which should be due to the insertion of $-\mathrm{CH}_{3}$ methyl groups into some of the $\mathrm{SiO}_{4}$ tetrahedra units. The incorporation of $-\mathrm{CH}_{3}$ groups into the first coordination sphere of a Si-atom would lead to a decrease in the positive effective charge on silicon due to lower electronegativity of the carbon atom compared to oxygen, resulting in the shift of the fine structure of the $\mathrm{SiL}_{2,3}$ absorption spectrum towards lower energy. This effect is also accompanied by a gradual decrease in the ratio of intensities of features $b^{\prime}-b$ and $a-a^{\prime}$ (1,9 for SAT and 1,5 for PECVD-1), relative to that for por- $\mathrm{SiO}_{2}$ (2.1). The same tendency was observed for the sequence $\mathrm{SiO}_{2}-\mathrm{Si}_{3} \mathrm{~N}_{4}-\mathrm{SiC}^{21}$ (in all these compounds, the Si atom can occupy only tetrahedrally coordinated sites). It has been found that with decreasing electronegativity of the nearest neighbors of the Si atom, i.e., with a decrease in the molecular field anisotropy of the nearest surroundings, the low energy shift of the features of the $\mathrm{SiL}_{2,3}$-absorption spectrum and the decrease in the intensity of features $b$ (associated 
with $\mathrm{p} \rightarrow \mathrm{p}$-like transitions in tetrahedron) and $c$ (connected with the influence of the molecular field of the surrounding atoms) are traced. It is plausible to assume that the gradual decrease in the electronegativity of the surroundings of the $\mathrm{Si}$ atom in SAT and PECVD-1 films is due to the insertion of methyl groups. Comparing the value of the energy shift with the literature values, ${ }^{22}$ we can conclude that the main structural unit of both studied OSG films is a $\mathrm{SiO}_{3}\left(\mathrm{CH}_{3}\right)$ tetrahedron with one methyl group inserted, and no significant contribution of tetrahedra with multiple methyl group insertion occurs. The presence of a considerable number of pristine $\mathrm{SiO}_{4}$ tetrahedra in the structure of both OSG films can also be traced from the observation of a shoulder $b$, in the OSG $\mathrm{SiL}_{2,3}$-absorption spectra at $108.2 \mathrm{eV}$, closely resembling the corresponding peak $b$ in the reference stoichiometric $\mathrm{SiO}_{2}$ spectra.

CK(1s)-absorption spectra of SAT and PECVD-1, measured by total electron yield, are shown in Fig. 2a. Since an adhesion layer constituted mainly by carbon atoms might be present on the surface of the studied samples, we measured X-ray reflection spectra of both OSG films at two different grazing incidence angles, $2^{\circ}$ and $4^{\circ}$. The depth of formation of reflected radiation is significantly different for $2^{\circ}$ and $4^{\circ}(6 \mathrm{~nm}$ and $16 \mathrm{~nm}$ ), respectively, according to the estimation using Henke constants, ${ }^{23,24}$ and therefore the contribution of the surface adhesion layer should be considerably smaller for spectra measured at $4^{\circ}$. However, the calculated CK-absorption spectra were almost indistinguishable for each sample; we can therefore assert that there are inessential or no adhesion layers on the surface of OSG films, and the presented CK-absorption spectra correspond to the distribution of electronic states of OSG films without contribution of an adhesion layer. This can be supported by the fact that OSG has poor adhesion properties in the as-deposited state without special surface treatment. ${ }^{25,26}$

The presented CK-absorption spectra of OSG films (Fig. 2a) demonstrate similar fine structural features. These features may be identified as the following three main areas: $a\left(\mathrm{C} 1 \mathrm{~s} \rightarrow \pi^{*}\right.$ transitions in $\mathrm{C}=\mathrm{C}$ ), $b$ (C 1s $\rightarrow \sigma^{*}$ transitions in $\mathrm{Si}-\mathrm{CH}_{3}$ ) and broad $\mathrm{D}$ bands (C 1s $\rightarrow \sigma^{*}$ transitions in different bonds of carbon), when considered together with the model spectra of tetramethyl-silane ${ }^{27}$ (TMS) $\mathrm{Si}\left(\mathrm{CH}_{3}\right)_{4}$, amorphous carbon (am-C) ${ }^{28}$ and highly ordered pyrolytic graphite (HOPG). ${ }^{29}$ Note that the $1 \mathrm{~s} \rightarrow \pi^{*}$ and $1 \mathrm{~s} \rightarrow \sigma^{*}$ resonances are characteristic of $\mathrm{sp}^{2}$ hybridization, and the $1 \mathrm{~s} \rightarrow \sigma^{*}$ resonance can only be realized in $\mathrm{sp}^{3}$.

Analysis of the most intense peak, $b$, reveals two low energy shoulders $b_{1}$ and $b_{x}$ in the spectra of OSG films. The main peak $b$ and shoulder $b_{1}$ correlate well with analogous features in the CK-absorption spectrum of the TMS molecule, ${ }^{27}$ which correspond to transitions from the $\mathrm{C} 1 \mathrm{~s}$ level to $3 \mathrm{p}$ and $3 \mathrm{~s}$ Rydberg states mixed with $\sigma^{*}{ }_{\mathrm{C}-\mathrm{H}}$ orbitals. ${ }^{30}$ Moreover, the $b_{1}-b$ features in the spectrum of TMS are followed by $\sigma^{*}$ shape resonance $D_{1}$ with a maximum at $290 \mathrm{eV}$, which is a signature of the carbon single bond with the Si atom in the case of TMS. As is known, ${ }^{30}$ the specific position of resonance $D_{1}$ (and other $\sigma^{*}$ shape resonances $D_{2}$ and $D^{*}$ ) depends strongly on the bond length. In the case of $\mathrm{C}-\mathrm{C}$ bond, which is shorter $(1.54 \AA)$ than $\mathrm{Si}-\mathrm{C}$ $(1.85 \AA),{ }^{31,32}$ the resonance $D_{1}$ lies higher in energy at $292.5 \mathrm{eV}$,
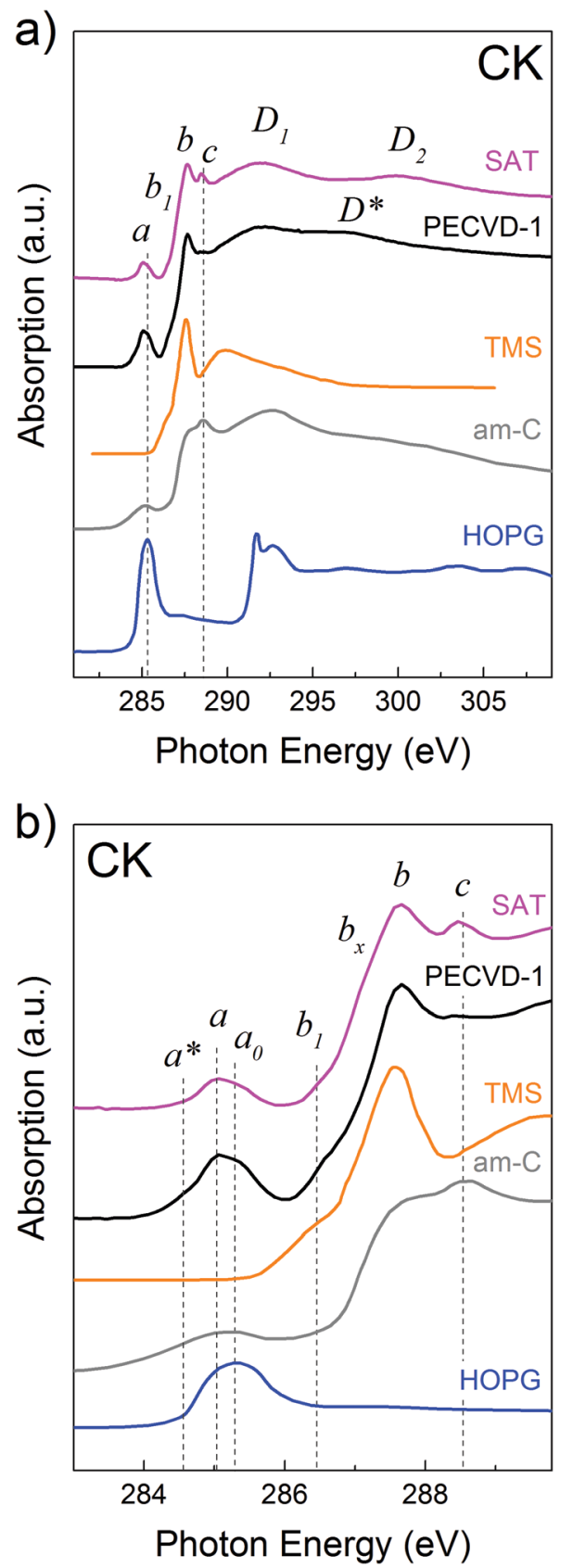

Fig. 2 (a) CK(1s)-absorption spectra of SAT and PECVD-1 measured by monitoring the total electron yield when the drain current from the sample is measured; tetramethyl-silane (TMS) $\mathrm{Si}\left(\mathrm{CH}_{3}\right)_{4}{ }_{4}{ }^{27}$ amorphous carbon ${ }^{28}$ and highly ordered pyrolytic graphite (HOPG). ${ }^{29}$ (b) Magnification of the spectra shown in panel (a) (the spectrum of HOPG is multiplied by 0.33 for visibility).

as can be seen for amorphous carbon in Fig. 2a. The resonance $D_{1}$ of both SAT and PECVD-1 films is located at $291.7 \mathrm{eV}$ and in this regard, it should be considered as a counterpart of features $b_{1}-b$, which are altogether the main spectral feature of OSG, indicating the presence of $\mathrm{Si}-\mathrm{CH}_{3}$ bonds.

The main maximum $a$ is located at $285.05 \mathrm{eV}$, which is followed by the shoulder $a_{0}$ at $285.3 \mathrm{eV}$ for both SAT and PECVD-1 (Fig. 2a and in detail in Fig. 2b). The shoulder $a_{0}$ 
corresponds to a single peak at the same energy observed in the spectrum of HOPG, which is due to $\mathrm{C} 1 \mathrm{~s} \rightarrow \pi_{\mathrm{C}=\mathrm{C}}^{*}$ transitions in carbon being in an ordered structure. The lower energy shift of the $a_{0}$ peak (maximum $a$ at $285.05 \mathrm{eV}$ in the spectra of OSG films) could be due to the formation of $\mathrm{sp}^{2}$ chains or isolated $\mathrm{sp}^{2}$ pairs of carbon atoms. ${ }^{33}$ Considering the shape of the entire peak $a$, we can conclude that the structure of PECVD-1 and SAT contains chains or isolated $\mathrm{sp}^{2}$ pairs of carbon atoms (maximum $a$ ) with some contribution of graphite-like rings (the shoulder $a_{0}$ ). From the intensity of this peak (in the case of SAT the peak is considerably smaller in intensity), one can conclude that the SAT method allows the synthesis of OSG films almost without $\mathrm{sp}^{2}$ carbon clusters. ${ }^{4}$ In the case of PECVD-1, the entire peak $a$ should be a signature of porogen residues in its structure; ${ }^{4}$ besides, the CK-absorption spectrum of PECVD-1 contains the low energy shoulder $a^{*}$ at $284.5 \mathrm{eV}$, which is accompanied by the shape resonance $D^{*}$ at $297 \mathrm{eV}$ (both features are absent in the spectrum of SAT). According to literature, ${ }^{30,34}$ the shoulder $a^{*}$ and shape resonance $D^{*}$ can be attributed to transitions in the $\pi^{*}$ and $\sigma^{*}$ states for delocalized $\mathrm{C}=\mathrm{C}$ bonds, which are characteristic of aromatic structures inherent in porogen molecules. It is worth noting that the general intensity of the entire peak $a$ is significantly smaller than the intensity of peak $b$ for both SAT and PECVD-1. This means that although some $\mathrm{sp}^{2}$ carbon clusters are present in the structure, the main state of carbon in OSG films is $\mathrm{sp}^{3}$ in $-\mathrm{CH}_{3}$ methyl groups bonded to $\mathrm{Si}$ atoms.

Special attention should be paid to the manifestation of feature $c$ and shoulder $b_{x}$. Both features are observed in the spectrum of SAT and only a trace can be found in the spectrum of PECVD-1. A joint analysis of these spectra with the spectrum of am-C reveals that feature $c$ and shoulder $b_{x}$ are related to permitted $\mathrm{C} 1 \mathrm{~s} \rightarrow \pi^{*}$, transitions induced by the presence of oxygen, and can be assigned to $\mathrm{C} 1 \mathrm{~s} \rightarrow \pi^{*} \mathrm{O}=\mathrm{C}$ (shoulder $b_{x}$ ) and $\mathrm{C} 1 \mathrm{~s} \rightarrow \pi^{*} \mathrm{O}=\mathrm{C}-\mathrm{OH}$ transitions (feature $c$ ). ${ }^{28,30,35,36}$ This assignment finds its principal justification in the anti-correlation of the intensity of features $a$ and $c-b_{x}$ (the more expressed features $c-b_{x}$ the less peak $a$ ), which shows the reduced $\mathrm{C}=\mathrm{C} \mathrm{sp}^{2}$ fraction in the spectrum of SAT due to the increment of hydrogen or $\mathrm{O}=\mathrm{C}-\mathrm{OH}$ acidic functional groups as peripheral termination of the graphitic domains. The $\sigma^{*}$ shape resonance $D_{2}$ at $300 \mathrm{eV}$ may be considered as a counterpart of the $\pi^{*}{ }_{\mathrm{C}}=\mathrm{O}$ state.

Fig. 3 shows the OK(1s)-absorption spectra of $\mathrm{am}_{-} \mathrm{SiO}_{2}$, por-SiO SAT and PECVD-1 films measured by total electron yield. The spectrum of $\alpha$-quartz was obtained previously. ${ }^{37}$ According to MS-X $\alpha$ calculations, ${ }^{37}$ the main absorption band $a-b$ in the OKabsorption spectrum of $\alpha$-quartz originates from the transitions of $\mathrm{O} 1 \mathrm{~s}$ electrons to $\mathrm{O} 2 \mathrm{p}$ states mixed with $\mathrm{Si} 3 \mathrm{~s}, 3 \mathrm{p}$ states. The features $c$ and $d$ originate from transitions of $\mathrm{O} 1 \mathrm{~s}$ electrons to $\mathrm{O} 2 \mathrm{p}$ states mixed with Si $3 \mathrm{~d}$ states. The features $c$ and $d$ are a signature of the presence of long-range order in the $\alpha$-quartz crystal and appear due to the photoelectron wave scattering on the long coordinated spheres. Both these features are absent in the absorption spectra for $\mathrm{am}-\mathrm{SiO}_{2}$ and por-SiO${ }_{2}$.

A joint analysis of the OK-absorption spectra of $\alpha$-quartz, am-SiO ${ }_{2}$ and por-SiO ${ }_{2}$ reveals the appearance of a low energy

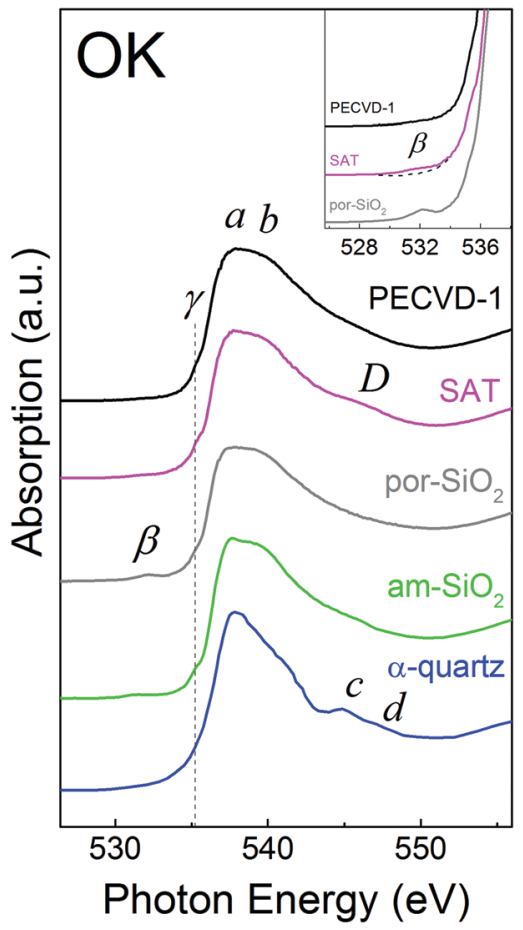

Fig. $3 \mathrm{OK}(1 \mathrm{~s})$-absorption spectra of $\mathrm{am}-\mathrm{SiO}_{2}$, por- $\mathrm{SiO}_{2}, \mathrm{SAT}$ and PECVD-1 films measured by monitoring the total electron yield when the drain current from the sample was measured; the $\alpha$-quartz spectrum was obtained from literature. ${ }^{37}$ The inset shows the pre-edge region of the last three spectra.

shoulder $\gamma$ at $535 \mathrm{eV}$ in the spectra of am-SiO ${ }_{2}$ and por-SiO${ }_{2}$, as compared with the spectrum of $\alpha$-quartz. A peak $\beta$ located at $532.2 \mathrm{eV}$ is traced in the pre-edge region of the spectrum of por- $\mathrm{SiO}_{2}$. All these features were formed as a result of the presence of some dangling $\mathrm{Si}-\mathrm{O}$ bonds in both structures. Besides, one can observe a gradual decrease in the intensity of feature $b$, relative to the intensity of feature $a$ in the sequence por- $\mathrm{SiO}_{2}-\mathrm{am}-\mathrm{SiO}_{2}-\alpha$-quartz. According to literature, ${ }^{38}$ such redistribution of relative intensities of features $a$ and $b$ may be related to some reduction in the degree of mixing $\mathrm{Si} 3 \mathrm{p}-\mathrm{O} 2 \mathrm{p}$ orbitals due to the elongation of $\mathrm{Si}-\mathrm{O}$ bonds as a result of gradual structure disordering in am- $\mathrm{SiO}_{2}$ and por- $\mathrm{SiO}_{2}$.

As observed from the Fig. 3, the spectra of the studied SAT and PECVD-1 films are similar to the spectrum of por-SiO ${ }_{2}$. A formation of the pre-edge region of the OK-absorption spectra of the studied OSG films (inset in Fig. 3) draws some attention. There is no peak $\beta$ in the spectrum of PECVD- 1 and only a slight hint can be observed in that of the SAT sample, indicating the absence of dangling Si-O bonds in the structure of SAT and PECVD-1, which may be passivated by $-\mathrm{CH}_{3}$ methyl groups. Moreover, the shoulder $\gamma$ was enhanced without significantly changing the shape of the main absorption band $a-b$ in the spectra of SAT and PECVD-1, which can be related to additional negative charge transfer to oxygen atoms in $\mathrm{SiO}_{4-n}\left(\mathrm{CH}_{3}\right)_{n}$ tetrahedra, due to the substitution of oxygen by $\left(-\mathrm{CH}_{3}\right)$ methyl groups. An additional broad feature $D$ is present in the spectrum of SAT at around $546 \mathrm{eV}$. This feature originates from $\mathrm{O}$ 1s $\rightarrow \sigma^{*} \mathrm{O}=\mathrm{C}-\mathrm{OH}$ transitions, due to the presence of 
carboxyl groups ${ }^{35}$ in the structure of SAT, which were also revealed in the CK-absorption spectrum.

In order to align the measured $\mathrm{SiL}_{2,3^{-}}, \mathrm{CK}$ and OK-absorption spectra in a common energy scale, the Si 2 p, C 1s and O 1s photoelectron spectra were measured under the same experimental conditions at $700 \mathrm{eV}$ excitation energy; the spectra of SAT, PECVD-1 and am-SiO ${ }_{2}$ are plotted in Fig. 4a-c. The energy position of the $\mathrm{Si} 2 \mathrm{p}$ line (Fig. 4a) of am- $\mathrm{SiO}_{2}$ is $103.3 \mathrm{eV}$, which correlates with values reported for $\mathrm{SiO}_{2}$ in literature. ${ }^{39,40}$ One can see that the Si 2p lines of SAT and PECVD-1 are shifted to lower binding energy by $0.25 \mathrm{eV}$ and $0.4 \mathrm{eV}$, respectively; besides, the full width of the Si $2 \mathrm{p}$ line of SAT and PECVD-1 gradually increases relative to that of am-SiO ${ }_{2}$. This can be attributed to the formation of an additional component related to excitation from the Si atom, which is surrounded by neighbors with lower electronegativity. As stated in the literature, ${ }^{41,42}$ new components should be related to the formation of $\mathrm{SiO}_{3}\left(\mathrm{CH}_{3}\right)$ tetrahedra with one methyl group, according to its energy position near $102.5 \mathrm{eV}$. We can also conclude that the insertion of methyl groups is more pronounced for PECVD-1, according to the greater energy shift and increase of the full width of the Si $2 p$ line.

C 1s spectra of SAT, PECVD-1 and $\mathrm{am}-\mathrm{SiO}_{2}$ are shown in Fig. $4 \mathrm{~b}$. It should be stressed that as was mentioned in the Experimental section, the surface of OSG was free of any adhesion layer. The shapes of the spectra of SAT and PECVD-1 are nearly the same, with a single main peak at $284.6 \mathrm{eV}$, which should be assigned to the $\mathrm{Si}-\mathrm{CH}_{3}$ bond. Although the main peak

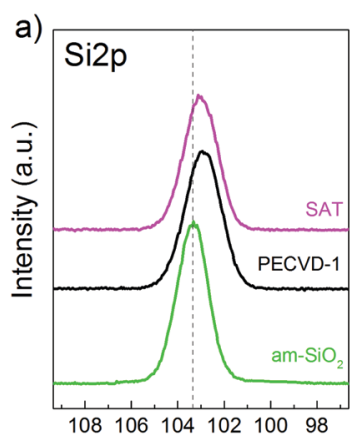

Binding Energy (eV)

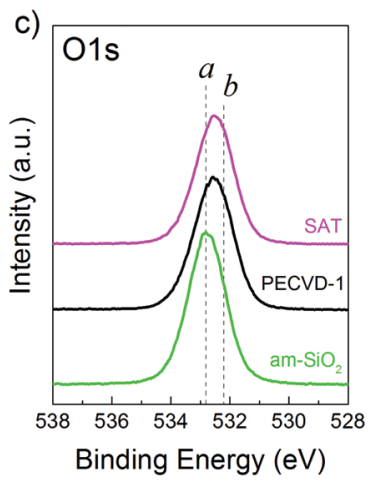

Fig. 4 Si $2 p$ (a), C 1s (b) and O 1s (c) XPS spectra of SAT, PECVD-1 and am- $-\mathrm{SiO}_{2}$ measured at the excitation energy of $700 \mathrm{eV}$, using the charge neutralization system. The inset in panel (b) shows the area of peak $c$ in the $C$ 1s spectra. at $284.6 \mathrm{eV}$ should contain some contribution of $\mathrm{sp}^{2}$ carbon clusters, as revealed from the analysis of CK-absorption spectra, we believe that this peak was primarily formed due to $\mathrm{Si}_{-} \mathrm{CH}_{3}$ bonds. This can be supported by analysis of the CKLL Auger band (not shown) of SAT and PECVD-1. The values of the $D$-parameter ${ }^{43}$ extracted from the derivative of the CKLL Auger band are equal to $14 \mathrm{eV}$ for PECVD-1, which is close to almost $100 \%$ content of carbon in $\mathrm{sp}^{3}$ state, and $16 \mathrm{eV}$ for SAT, indicating some contribution of carbon in the $\mathrm{sp}^{2}$ state. Bearing in the mind the minimal intensity of peak $a$ in the CK-absorption spectrum of SAT, we can assert that most $\mathrm{sp}^{2}$ carbon atoms form $\mathrm{O}=\mathrm{C}-\mathrm{OH}$ bonds. The spectrum of SAT contains a slight peak $c$ at $289 \mathrm{eV}$, shown in the inset in Fig. 4b. This peak can be related to the presence of carboxyl groups ${ }^{44,45}$ in the structure of SAT, which were revealed by the analysis of the CK-absorption spectra. Finally, one can see that the shape of the $\mathrm{C} 1 \mathrm{~s}$ spectrum of $\mathrm{am}-\mathrm{SiO}_{2}$, due to surface contamination, significantly differs from that of SAT and PECVD-1. This fact supports our conclusion about the absence of surface contamination of OSG films.

O 1s spectra of SAT, PECVD-1 and am-SiO ${ }_{2}$ are shown in Fig. 4c. The peak $a$ at $532.7 \mathrm{eV}$ correlates well with that of $\mathrm{SiO}_{2} \cdot{ }^{39}$ The $\mathrm{O}$ 1s spectra of SAT and PECVD-1 are shifted to lower binding energy due to the formation of a new component $b$, which we assign to the formation of $\mathrm{SiO}_{3}\left(\mathrm{CH}_{3}\right)$ tetrahedra. According to ref. 46-48 the peaks corresponding to the $\mathrm{O}=\mathrm{C}-\mathrm{OH}$ and $\mathrm{O}=\mathrm{C}$ groups overlap with the main $a-b$ peak. Accounting for the small intensity of the corresponding $\mathrm{C} 1 \mathrm{~s}$ peak $c$ in the spectrum of SAT (assigned to $\mathrm{O}=\mathrm{C}-\mathrm{OH}$ groups and being the only peak intense enough to resolve), it is not possible to resolve oxygenated carbon peaks in the $\mathrm{O} 1 \mathrm{~s}$ spectrum of SAT.

Fig. 5a shows the $\mathrm{SiL}_{2,3^{-}}$, CK- and OK-absorption spectra of SAT, PECVD-1, por-SiO ${ }_{2}$, am-SiO ${ }_{2}$ and $\alpha$-quartz on a common energy scale, where the Fermi level is chosen as the zero point of the energy scale; the valence band is also shown. The correct combination was achieved with help of XPS data. In the XPS method, it is assumed that there is a thermodynamic equilibrium in the system "sample-spectrometer" so that the Fermi levels of a sample and spectrometer are equalized. Then, if the binding energies $\left(E_{\mathrm{B}}\right)$ of a given state are measured from common Fermi level, the energy conservation is as follows:

$$
h \nu=E_{\mathrm{B}}+\varphi+E_{\mathrm{kin}}
$$

where $h \nu$ is the energy of the X-ray photon, $\varphi$ is the photoelectric work function, and $E_{\text {kin }}$ is the kinetic energy of the electrons ejected from the $E_{\mathrm{B}}$ level. Eqn (1) allows the calculation of the binding energies $E_{\mathrm{B}}$ of all of the electrons participating in the photo effect based on measured $E_{\text {kin }}$. The binding energies, obtained from the XPS spectra (Fig. 4) were used to compare the X-ray absorption spectra of different atoms constituting the film on the common energy scale. The Fermi level was chosen as the zero-point of the energy scale.

One can see that all the presented OK-absorption spectra correlate well in the position of the OK-absorption edge, which could be associated with the bottom of a conduction band $\mathrm{E}_{\mathrm{C}}$. Extraction of the $\mathrm{E}_{\mathrm{C}}$ position from $\mathrm{SiL}_{2,3}$ and the CK-absorption spectra is complicated by different localized states under $\mathrm{E}_{\mathrm{C}}$. In 

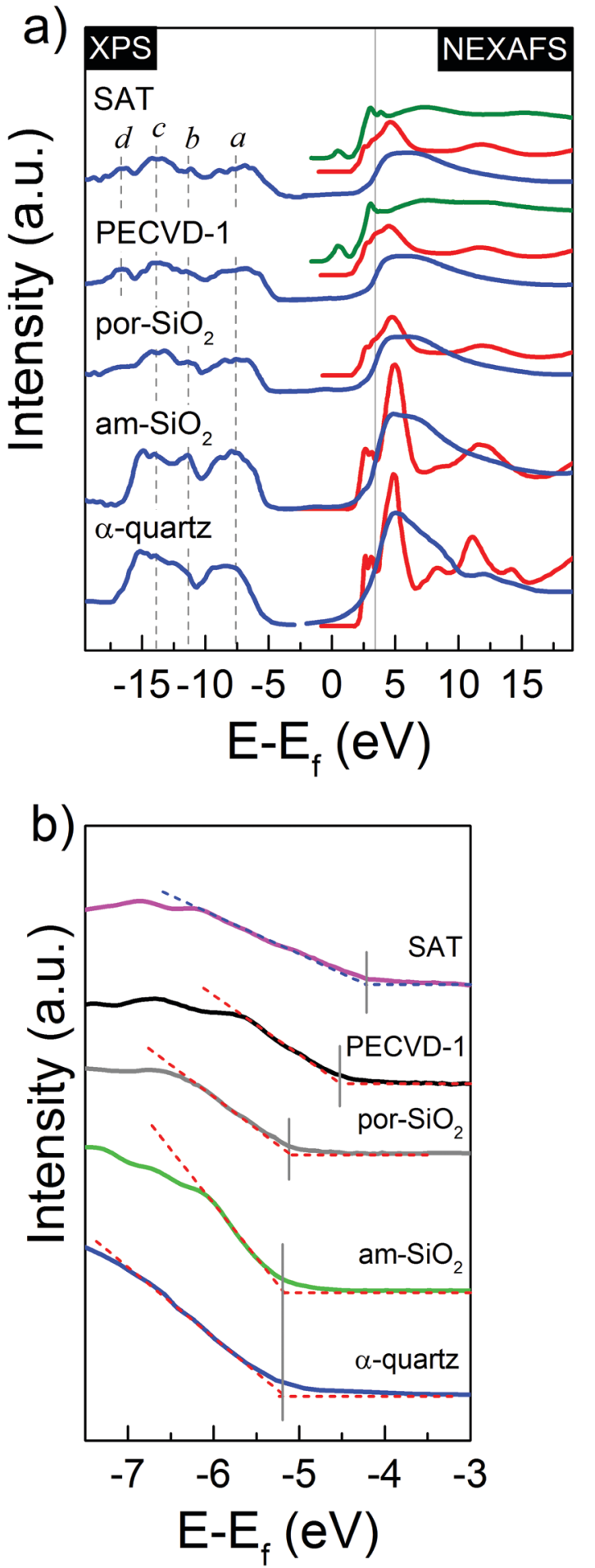

Fig. 5 (a) OK- (blue), $\mathrm{SiL}_{2,3^{-}}$(red) and $\mathrm{CK}$ - (green) absorption spectra and spectra of the valence band measured at $1486.6 \mathrm{eV}$ excitation energy (blue) for SAT, PECVD-1, por- $\mathrm{SiO}_{2}, \mathrm{am}-\mathrm{SiO}_{2}$ and $\alpha$-quartz on a common energy scale, where the Fermi level is chosen as the zero-point of the energy scale. (b) Magnification of the top of the valence band of the spectra shown in panel (a).

this case, the value of the band gap can be extracted for am- $-\mathrm{SiO}_{2}$ to be equal to $8.7 \mathrm{eV}$, which correlates well with literature values. ${ }^{49,50}$ Thus, we can conclude from the coincidence of
OK-edge positions that introducing porosity in the structure of $\mathrm{SiO}_{2}$ and insertion of methyl groups in $\mathrm{SiO}_{4}$ tetrahedra do not change the position of $\mathrm{E}_{\mathrm{C}}$. The overlapping of the main absorption band $a-b$ in the OK-absorption spectra with feature $b$ in the $\mathrm{SiL}_{2,3}$-absorption spectra can clearly be observed and is due to strong overlap between Si 3p-orbitals and O 2p-orbitals in the tetrahedral coordination of Si atoms. The doublet feature $a-a^{\prime}$ (assigned to transitions from the Si 2p states to the Si 3s states) in the $\mathrm{SiL}_{2,3}$-absorption spectra of all the studied samples is located under the $\mathrm{E}_{\mathrm{C}}$ and can be interpreted to be due to the creation of an exciton localized on the silicon atom. ${ }^{51}$

As was mentioned above, the low energy shift of the features of the $\mathrm{SiL}_{2,3}$-absorption spectra (both the main features $a$ and $b$ ) of OSG occurs. As can be seen from Fig. $5 a$ accounting for the binding energy of the initial Si $2 p$ level, the energy shift preserves only feature $b^{\prime}$ in the spectra of SAT and PECVD-1, with features $a-a^{\prime}$ not being shifted relative to that in the $\mathrm{SiL}_{2,3^{-}}$ absorption spectra of am- $-\mathrm{SiO}_{2}$ and $\alpha$-quartz. This means that only feature $b^{\prime}$ is shifted due to the shift of the final molecular orbital $t_{2}$ because of the change in the electronegativity of the surroundings of the absorbing atom (insertion of methyl groups). Thus, the energy shift of features $a-a^{\prime}$ traced in $\mathrm{SiL}_{2,3}$-absorption spectra shown in Fig. 1 is due to the change the binding energy of the initial Si 2 p level only. The analogous effect was observed for monolayers of $\mathrm{SiO}_{2}$ compared with bulk $\mathrm{SiO}_{2}$ due to significant variation in the $\mathrm{Si}-\mathrm{O}-\mathrm{Si}$ valence angles. ${ }^{51}$ In our case, this effect can be attributed to the formation of high level porosity in OSG films.

Finally, one can find peak $a$ in the CK-absorption spectra of SAT and PECVD-1, which is due to C $1 \mathrm{~s} \rightarrow \pi^{*} \mathrm{C}=\mathrm{C}$ transitions, near the Fermi energy. As was shown in the literature, ${ }^{4}$ PECVD-1 is characterized by a significant leakage current. The analysis of absorption spectra aligned in a common energy scale (Fig. 5) allows us to conclude that particularly the peak $a$ in the CK-absorption spectra is responsible for the leakage current.

It should be noted that Fig. 5 shows the photoelectron spectra of the valence band of all the studied samples measured at an excitation energy of $1486.6 \mathrm{eV}$. According to the calculation of the inelastic mean free path based on the TPP-2M formula,${ }^{14}$ the probing depth of the XPS spectra of the VB measured at $1486.6 \mathrm{eV}$ excitation energy was comparable to that for the measured absorption spectra. Note that the XPS spectra of the core levels measured at $1486.6 \mathrm{eV}$ excitation energy correlated well with those measured at $700 \mathrm{eV}$ with respect to shape and energy positions.

The spectra of SAT and PECVD-1 correlate well, in terms of the number and energy position of features $a, b$ and $c$, with the spectrum of $\mathrm{am}-\mathrm{SiO}_{2}$, which reflects $\mathrm{O} 2 \mathrm{p}$ nonbonding states and $\mathrm{O} 2 \mathrm{p}$ mixed with Si $3 \mathrm{p}$ and Si $3 \mathrm{~s}$ states, respectively. ${ }^{52-54}$ The feature $d$ in the spectra of SAT and PECVD- 1 is due to the $\mathrm{C} 2 \mathrm{~s}$ valence states of inserted methyl groups. The most important aspect is to analyze the position of $E_{V}$, which is shown in Fig. 5b. One can see that $\mathrm{E}_{\mathrm{V}}$ of $\mathrm{am}-\mathrm{SiO}_{2}$ is located at $5.2 \pm 0.1 \mathrm{eV}$, and $\mathrm{E}_{\mathrm{V}}$ of por- $\mathrm{SiO}_{2}$ is slightly shifted to $5.0 \pm 0.1 \mathrm{eV}$, while the $\mathrm{E}_{\mathrm{V}}$ of PECVD-1 is shifted to $4.5 \pm 0.1 \mathrm{eV}$. Such a large shift for PECVD-1 can be attributed to the insertion of methyl groups in $\mathrm{SiO}_{4}$ tetrahedra, while porosity does not significantly affect the $E_{V}$ 


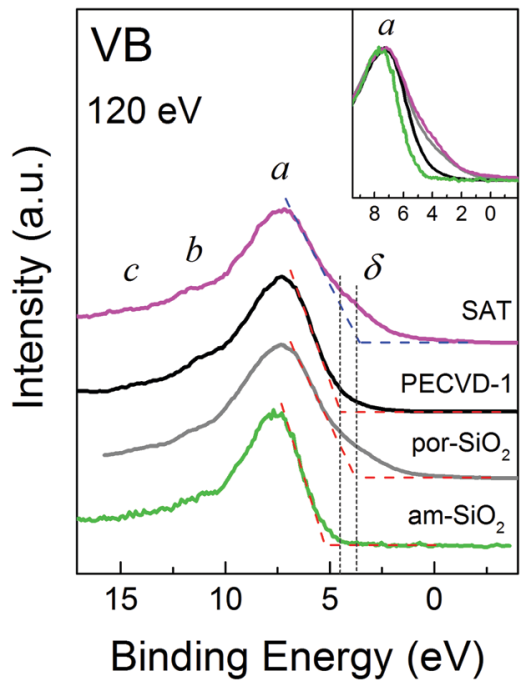

Fig. 6 VB XPS spectra of SAT, PECVD-1, por- $-\mathrm{SiO}_{2}$ and $\mathrm{am}-\mathrm{SiO}_{2}$ measured at excitation energy of $120 \mathrm{eV}$.

position, according to a slight $0.2 \mathrm{eV}$ shift for por-SiO ${ }_{2}$. One can see that the $\mathrm{E}_{\mathrm{V}}$ of the SAT sample is further shifted to $4.2 \pm 0.1 \mathrm{eV}$. Based on the presented analysis of absorption and photoelectron spectra, we can attribute such a shift in $\mathrm{E}_{\mathrm{V}}$ of SAT to the presence of $\mathrm{O}=\mathrm{C}-\mathrm{OH}$ and $\mathrm{C}=\mathrm{O}$ bonds in its structure.

The VB spectra of SAT, PECVD-1, por-SiO ${ }_{2}$ and $\mathrm{am}_{-} \mathrm{SiO}_{2}$ were also measured at $120 \mathrm{eV}$ excitation energy (Fig. 6), which provided extreme surface sensitivity. Due to the redistribution of photoabsorption cross-section values for different valence states, depending on photon energy, the shapes of the VB spectra were significantly different, compared to those measured at $1486.6 \mathrm{eV}$ excitation. The $\mathrm{E}_{\mathrm{V}}$ positions for the spectra of $\mathrm{am}-\mathrm{SiO}_{2}$ and PECVD-1 correlated well with that measured at $1486.6 \mathrm{eV}$ excitation. Nevertheless, the $\mathrm{E}_{\mathrm{V}}$ of SAT and por-SiO ${ }_{2}$ were shifted to $3.8 \pm 0.1 \mathrm{eV}$ and a shoulder $\delta$ appeared. Accounting for extreme surface sensitivity in the case of $120 \mathrm{eV}$ excitation, we can ascribe the additional energy shift of $\mathrm{E}_{\mathrm{V}}$ for SAT and por-SiO $\mathrm{S}_{2}$ to the enhanced oxidation of carbon at the surface (adventitious in case of por-SiO${ }_{2}$ ). The most plausible explanation of the shoulder $\delta$ appearing from the smaller binding energy side is the presence of dangling $\mathrm{Si}-\mathrm{O}$ bonds in the surface sublayers of SAT and por-SiO ${ }_{2}$. Analysis of the OK-absorption spectra of por- $\mathrm{SiO}_{2}$ and SAT reveals a formation of peak $\beta$ in the pre-edge region of the spectrum of por- $\mathrm{SiO}_{2}$, which points to the presence of some number of dangling $\mathrm{Si}-\mathrm{O}$ bonds. Moreover, a weak trace of peak $\beta$ in the spectrum of SAT can be seen. Accounting for the weak intensity of peak $\beta$ in the OK-absorption spectrum of SAT (inset in Fig. 3), and the absence of the shoulder $\delta$ in the VB XPS spectra of both por-SiO and SAT measured at $1486.6 \mathrm{eV}$ (Fig. 5b), we can conclude that only a small number of $\mathrm{Si}-\mathrm{O}$ dangling bonds were present at the very surface of SAT and a considerably greater number was present in some deeper layers of por-SiO ${ }_{2}$.

\section{Conclusions}

The formation of valence and conduction band states of $\mathrm{SiO}_{2}$ was studied depending on the introduction of porosity and the insertion of methyl groups into its structure. It was found that the bottom of the conduction band was not affected by introducing porosity and changing the electronegativity of the nearest surrounding neighbors of $\mathrm{Si}$ atoms. On the contrary, the top of valence band for PECVD-1 film was shifted by $0.7 \mathrm{eV}$ to the lower binding energy, due to the insertion of methyl groups, and this effect was further enhanced for SAT film, due to the presence of $\mathrm{O}=\mathrm{C}-\mathrm{OH}$ and $\mathrm{C}=\mathrm{O}$ bonds in its structure. Introducing porosity does not significantly change the position of $\mathrm{E}_{\mathrm{V}}$. The $\pi^{*} \mathrm{C}=\mathrm{C}$ state was found near the Fermi energy for both OSG films, which was more pronounced for traditional PECVD-1 film. This state is particularly responsible for the leakage current in PECVD-1 film. The significant variation in $\mathrm{Si}-\mathrm{O}-\mathrm{Si}$ valence angles due to high levels of porosity in OSG films was established from the low energy shifting of the first $a-a^{\prime}$ excitonic states localized on Si atoms. Changing the electronegativity of the nearest surrounding neighbors of $\mathrm{Si}$ atoms leads to the energy shift of the second main feature $b$ in the $\mathrm{SiL}_{2,3}$ absorption spectra of OSG films. A number of Si-O dangling bonds were found in the surface layers of por- $\mathrm{SiO}_{2}$, and only a small number of those were present at the very surface of SAT.

\section{Conflicts of interest}

There are no conflicts of interest to declare.

\section{Acknowledgements}

We gratefully acknowledge the assistance from the bilateral Program "Russian-German Laboratory" at HZB BESSY II and thank Dmitry Smirnov for valuable assistance. We gratefully acknowledge the financial support by Helmholtz Zentrum Berlin (HZB) and also thank HZB for the allocation of synchrotron radiation beamtime. The authors gratefully also acknowledge the employees of the Centre "Physical methods of surface investigation" of the Research park of St. Petersburg State University. This work was supported by RFBR grant 16-32-00276 mol_a.

\section{References}

1 A. Grill, S. M. Gates, T. E. Ryan, S. V. Nguyen and D. Priyadarshini, Appl. Phys. Rev., 2014, 1, 011306.

2 K. Maex, M. R. Baklanov, D. Shamiryan, F. Iacopi, S. H. Brongersma and Z. S. Yanovitskaya, J. Appl. Phys., 2003, 93, 8793-8841.

3 E. O. Filatova and A. S. Konashuk, J. Phys. Chem. C, 2015, 119, 20755-20761.

4 A. Konashuk, E. Filatova, S. Sakhonenkov and V. Afanas'ev, Microelectron. Eng., 2017, 178, 209-212.

5 V. S. Kortov, A. F. Zatsepin, S. V. Gorbunov and A. M. Murzakaev, Phys. Solid State, 2006, 48, 1273-1279.

6 L. E. Scriven, Mater. Res. Soc. Symp. Proc., 1988, 121, 717.

7 E. A. Smirnov, K. Vanstreels, P. Verdonck, I. Ciofi, D. Shamiryan, M. R. Baklanov and M. Phillips, Jpn. J. Appl. Phys., 2011, 50, 05EB03. 
8 V. Jousseaume, A. Zenasni, O. Gourhant, L. Favennec and M. R. Baklanov, in Advanced Interconnects for ULSI Technology, ed. M. R. Baklanov, P. Ho, E. Zschech, Wiley, Chichester, U.K., 1st edn, 2012, ch. 2, pp. 35-78.

9 S. I. Fedoseenko, I. E. Iossifov, S. A. Gorovikov, J.-H. Schmidt, R. Follath, S. L. Molodtsov, V. K. Adamchuk and G. Kaindl, Nucl. Instrum. Methods Phys. Res., Sect. A, 2001, 470, 84-88.

10 M. P. Seah, Surf. Interface Anal., 2001, 31, 721-723.

11 M. G. Helander, M. T. Greiner, Z. B. Wang and Z. H. Lu, Rev. Sci. Instrum., 2011, 82, 096107.

12 J. A. Wilks and J. A. Kelber, Appl. Surf. Sci., 2009, 255, 9543-9547.

13 M. A. Goldman, D. B. Graves, G. A. Antonelli, S. P. Behera and J. A. Kelber, J. Appl. Phys., 2009, 106, 013311.

14 S. Tanuma, C. J. Powell and D. R. Penn, Surf. Interface Anal., 1994, 21, 165-176.

15 F. Schäfers and A. Sokolov, J. Large Scale Res. Facil., 2016, 2, A50.

16 E. O. Filatova, A. S. Shulakov and V. A. Luk'yanov, Phys. Solid State, 1998, 40, 1237-1240.

17 E. Filatova, V. Luk'anov, R. Barchewitz, J.-M. André, M. Idir and Ph. Stemmler, J. Phys.: Condens. Matter, 1999, 11, 3355-3370.

18 E. O. Filatova, A. A. Sokolov, J.-M. André, F. Schaefers and W. Braun, Appl. Opt., 2010, 49, 2539-2546.

19 D. Li, G. M. Bancroft, M. Kasrai, M. E. Fleet, R. A. Secco, X. H. Feng, K. H. Tan and B. X. Yang, Am. Mineral., 1994, 79, 622-632.

20 I. Tanaka, J. Kawai and H. Adachi, Phys. Rev. B: Condens. Matter Mater. Phys., 1995, 52, 11733-11739.

21 E. O. Filatova, A. S. Vinogradov and T. M. Zimkina, Phys. Solid State, 1985, 27, 606-609.

22 D. G. J. Sutherland, M. Kasrai, G. M. Bancroft, Z. F. Liu and K. H. Tan, Phys. Rev. B: Condens. Matter Mater. Phys., 1993, 48, 14989-15001.

23 X-Ray Interactions With Matter, http://henke.lbl.gov/opti cal_constants/.

24 B. L. Henke, E. M. Gullikson and J. C. Davis, At. Data Nucl. Data Tables, 1993, 54, 181-342.

25 A. Grill, D. Edelstein, M. Lane, V. Patel, S. Gates, D. Restaino and S. Molis, J. Appl. Phys., 2008, 103, 054104.

26 F. Iacopi, S. H. Brongersma, B. Vandevelde, M. O’Toole, D. Degryse, Y. Travaly and K. Maex, Microelectron. Eng., 2004, 75, 54-62.

27 S. G. Urquhart, J. Z. Xiong, A. T. Wen, T. K. Sham, K. M. Baines, G. G. B. de Souza and A. P. Hitchcock, Chem. Phys., 1994, 189, 757-768.

28 C. Lenardi, P. Piseri, V. Briois, C. E. Bottani, A. Li Bassi and P. Milani, J. Appl. Phys., 1999, 85, 7159-7167.

29 P. E. Batson, Phys. Rev. B: Condens. Matter Mater. Phys., 1993, 48, 2608-2610.

30 A. P. Hitchcock, S. Beaulieu, T. Steel, J. Stöhr and F. Sette, J. Chem. Phys., 1984, 80, 3927-3935.

31 T. L. Cottrell, The Strengths of Chemical Bonds, Butterworths Publications Ltd, London, U.K., 1958.
32 B. deB. Darwent, National Standard Reference Data Series, National Bureau of Standards, Washington, USA, 1970.

33 R. Gago, M. Vinnichenko, H. U. Jäger, A. Yu. Belov, I. Jiménez, N. Huang, H. Sun and M. F. Maitz, Phys. Rev. B: Condens. Matter Mater. Phys., 2005, 72, 014120.

34 J. Diaz, S. Anders, X. Zhou, E. J. Moler, S. A. Kellar and Z. Hussain, Phys. Rev. B: Condens. Matter Mater. Phys., 2001, 64, 125204.

35 D. A. Outka, J. Stöhr, R. J. Madix, H. H. Rotermund, B. Hermsmeier and J. Solomon, Surf. Sci., 1987, 185, 53-74.

36 M. Jaouen, G. Tourillon, J. Delafond, N. Junqua and G. A. Hug, Diamond Relat. Mater., 1995, 4, 200-206.

37 Z. Y. Wu, F. Jollet and F. Seifert, J. Phys.: Condens. Matter, 1998, 10, 8083-8092.

38 H. M. Tsai, S. C. Ray, C. W. Pao, J. W. Chiou, C. L. Huang, C. H. Du, W. F. Pong, M.-H. Tsai, A. Fukano and H. Oyanagi, J. Appl. Phys., 2008, 103, 013704.

39 Th. Gross, M. Ramm, H. Sonntag, W. Unger, H. M. Weijers and E. H. Adem, Surf. Interface Anal., 1992, 18, 59-64.

40 F. J. Himpsel, F. R. McFeely, A. Taleb-Ibrahimi, J. A. Yarmoff and G. Hollinger, Phys. Rev. B: Condens. Matter Mater. Phys., 1988, 38, 6084-6096.

41 C. Onneby and C. G. Pantano, J. Vac. Sci. Technol., A, 1997, 15, 1597-1602.

42 B. Lanfant, Y. Leconte, G. Bonnefont, V. Garnier, Y. Jorand, S. Le Gallet, M. Pinault, N. Herlin-Boime, F. Bernard and G. Fantozzi, J. Eur. Ceram. Soc., 2015, 35, 3369-3379.

43 A. Mezzi and S. Kaciulis, Surf. Interface Anal., 2010, 42, 1082-1084.

44 Yu. V. Fedoseeva, G. A. Pozdnyakov, A. V. Okotrub, M. A. Kanygin, Yu. V. Nastaushev, O. Y. Vilkov and L. G. Bulusheva, Appl. Surf. Sci., 2016, 385, 464-471.

45 P. Hammer, F. C. dos Santos, B. M. Cerrutti, S. H. Pulcinelli and C. V. Santilli, Prog. Org. Coat., 2013, 76, 601-608.

46 Y. J. Oha, J. J. Yoo, Y. Il Kim, J. K. Yoon, H. N. Yoon, J.-H. Kim and S. B. Park, Electrochim. Acta, 2014, 116, 118-128.

47 R. Arrigo, M. Havecker, S. Wrabetz, R. Blume, M. Lerch, J. McGregor, E. P. J. Parrott, J. A. Zeitler, L. F. Gladden, A. Knop-Gericke, R. Schlogl and D. S. Su, J. Am. Chem. Soc., 2010, 132, 9616-9630.

48 C. Tao, J. Wang, S. Qin, Y. Lv, Y. Long, H. Zhu and Z. Jiang, J. Mater. Chem., 2012, 22, 24856-24861.

49 J. A. Kittl, K. Opsomer, M. Popovici, N. Menou, B. Kaczer, X. P. Wanga, C. Adelmann, P. A. Pawlak, K. Tomida and A. Rothschild, et al., Microelectron. Eng., 2009, 86, 1789-1795.

50 E. P. O'Reilly and J. Robertson, Phys. Rev. B: Condens. Matter Mater. Phys., 1983, 27, 3780-3795.

51 A. Bianconi, Surf. Sci., 1979, 89, 41-50.

52 S. S. Nekrashevich and V. A. Gritsenko, Phys. Solid State, 2014, 56, 207-222.

53 O. Yu. Khyzhun, T. Strunskus, Ch. Wöll, H. Gies and V. Staemmler, J. Chem. Phys., 2008, 129, 084711.

54 D. A. Zatsepin, P. Mack, A. E. Wright, B. Schmidt and H.-J. Fitting, Phys. Status Solidi A, 2011, 208, 1658-1661. 